\title{
Distance-Decay Relationships Partially Determine Diversity Patterns of Phyllosphere Bacteria on Tamrix Trees across the Sonoran Desert
}

\author{
Omri M. Finkel, ${ }^{\text {a }}$ Adrien Y. Burch, ${ }^{\text {b }}$ Tal Elad, $^{\text {a }}$ Susan M. Huse, ${ }^{c}$ Steven E. Lindow, ${ }^{\text {b }}$ Anton F. Post, ${ }^{c}$ and Shimshon Belkin ${ }^{a}$ \\ Institute of Life Sciences, Hebrew University of Jerusalem, Jerusalem, Israela; ; Department of Plant and Microbial Biology, University of California, Berkeley, California, USA ${ }^{\text {b; }}$ \\ and Josephine Bay Paul Center for Comparative Molecular Biology and Evolution, Marine Biology Laboratory, Woods Hole, Massachusetts, USA
}

\begin{abstract}
Dispersal limitation in phyllosphere communities was measured on the leaf surfaces of salt-excreting Tamarix trees, which offer unique, discrete habitats for microbial assemblages. We employed $16 \mathrm{~S}$ rRNA gene pyrosequencing to measure bacterial community dissimilarity on leaves of spatially dispersed Tamarix specimens in sites with uniform climatic conditions across the Sonoran Desert in the Southwestern United States. Our analyses revealed diverse bacterial communities with four dominant phyla that exhibited differential effects of environmental and geographic variables. Geographical distance was the most important parameter that affected community composition, particularly that of betaproteobacteria, which displayed a statistically significant, distance-decay relationship.
\end{abstract}

$E_{s}^{c}$ cological theories of biodiversity can be viewed from two perspectives (14). One, dubbed the "niche-assembly perspective" by Hubbell (14), holds that the biodiversity of a community is maintained by the partitioning of organisms to specialized niches within it, allowing the coexistence of large numbers of species in close proximity. The other is the "dispersal-assembly perspective," exemplified by the theory of island biogeography (22) and later by both the neutral theory of biogeography (14) and the metacommunity concept (21). This family of theories asserts that niche partitioning is not essential for the maintenance of biodiversity and that a community's history of stochastic dispersal and of random extinction and speciation events can explain much of the biodiversity observed in nature. While the latter perspective has gained much support in the ecology of macroorganisms, niche assembly theories have remained dominant in explaining the distribution of the most diverse organisms on earth, bacteria, as exemplified by the Baas-Becking hypothesis that "everything is everywhere, but the environment selects" (5). The main reason that niche partitioning is seen as the more important driver of microbial biodiversity is that dispersal-assembly requires a trait that most free living microbes seem to lack-a limitation on dispersal (23). Free-living microorganisms have the ability to disperse at seemingly infinite rates, rendering dispersal history an unimportant determinant in the assembly of a community, as the influx of immigrants should be sufficiently large to overshadow any stochastic effect. Investigations of dispersal limitation in the microbial world have recently become more frequent $(1,8,11,13)$. These studies typically measure dissimilarity in the community compositions of similar isolated environmental patches as a function of geographic distance. This measure, the "distance-decay relationship," is also the topic of the present study, in which we measure the distance-related relationships of bacteria in the phyllosphere of the salt-secreting desert tree Tamarix aphylla. One of the adaptations of Tamarix trees to surviving in deserts is to secrete solutes onto the leaf surface, rendering it extremely saline (35) and alkaline $(28,36)$. Microorganisms living on this surface are exposed to a stress "cocktail" consisting of elevated and fluctuating salinity, periodic desiccation, high temperatures, and UV radiation, and in some cases, high $\mathrm{pH}$. However, the Tamarix leaves provide an environment that is rich in organic carbon and is sufficiently moist to support a substantial epiphytic microbial population, even in extreme desert environments (28). While many Tamarix species are highly invasive, T. aphylla has limited sexual reproduction in the United States and, therefore, most $T$. aphylla stands are concentrated where they were planted decades ago. Tamarix trees spread throughout a desert may thus be considered analogous to an archipelago of islands: they offer a microbial habitat that is dramatically different from their immediate surroundings and support a metacommunity of microorganisms, the uniformity of which is influenced by, if not directly determined by, the rate of microbial dispersal between trees. The Tamarix phyllosphere is thus a particularly suitable system for study of the spatial scaling of microbial communities, since the trees exist as worldwide island-like microbial habitats.

In a recent report (9), we showed that the microbial composition of the Tamarix phyllosphere is dramatically different in the climates of the Dead Sea and Mediterranean coasts of Israel. In a different study (29), a distance-dependent dissimilarity was found between Tamarix phyllosphere populations within the Dead Sea region using denaturing gradient gel electrophoresis (DGGE) patterns. These findings indicated that dispersal limitation appears to play a role in the assembly of the microbial communities of the Tamarix phyllosphere. In the current communication, we put this hypothesis to further scrutiny by isolating geographical distance as the measured parameter. We have done this by sampling trees in a large desert area having relatively uniform climatic conditions, the Sonoran desert of the Southwestern United States. We sampled trees along a 500-km east-west transect and compared the diversity at the different sites by pyrosequencing the V4-to-V6 (V4-V6) hypervariable regions of the bacterial $16 \mathrm{~S}$ rRNA gene. We show that community dissimilarity is significantly correlated with geo-

Received 22 March 2012 Accepted 20 June 2012

Published ahead of print 29 June 2012

Address correspondence to Shimshon Belkin, shimshon.belkin@mail.huji.ac.il.

Supplemental material for this article may be found at http://aem.asm.org/

Copyright @ 2012, American Society for Microbiology. All Rights Reserved.

doi:10.1128/AEM.00888-12 
TABLE 1 Environmental variables ${ }^{a}$

\begin{tabular}{|c|c|c|c|c|c|c|c|c|}
\hline \multirow[b]{2}{*}{ Site } & \multirow[b]{2}{*}{ Altitude (m) } & \multirow[b]{2}{*}{$\mathrm{EC}(\mathrm{mS} / \mathrm{cm})$} & \multirow[b]{2}{*}{$\mathrm{pH}$} & \multirow[b]{2}{*}{$\mathrm{DOC}(\mathrm{mg} \mathrm{C} / \mathrm{g}$ leaf$)$} & \multicolumn{2}{|l|}{ Temp $\left({ }^{\circ} \mathrm{C}\right)$} & \multicolumn{2}{|c|}{ Humidity (\%) } \\
\hline & & & & & Maximum & Minimum & Maximum & Minimum \\
\hline $\mathrm{A} 1$ & 739 & 10.09 & 9.7 & 1.28 & 39 & 22 & 50.9 & 11.7 \\
\hline $\mathrm{A} 2$ & 739 & 12.53 & 8.08 & 1.81 & 39 & 22 & 50.9 & 11.7 \\
\hline B1 & 153 & 12.39 & 9.91 & 1.73 & 38 & 25 & 51.9 & 12 \\
\hline B2 & 153 & 8.47 & 9.89 & 1.13 & 38 & 25 & 51.9 & 12 \\
\hline $\mathrm{C} 1$ & 227 & 5.75 & 9.72 & 1.71 & 40 & 23 & 31.5 & 8.6 \\
\hline $\mathrm{C} 2$ & 227 & 15.84 & 7.79 & 2.48 & 40 & 23 & 31.5 & 8.6 \\
\hline D1 & 282 & 3.1 & 10.11 & 1.52 & 40 & 21 & 34.7 & 8.9 \\
\hline D2 & 282 & 2.08 & 8.24 & 0.78 & 40 & 21 & 34.7 & 8.9 \\
\hline E1 & 609 & 4.14 & 8.36 & 1.33 & 39 & 19 & 22.9 & 8.9 \\
\hline E2 & 677 & 5.49 & 8.67 & 1.54 & 39 & 19 & 22.9 & 8.9 \\
\hline
\end{tabular}

${ }^{a}$ Electrical conductivity (EC), $\mathrm{pH}$, and dissolved organic carbon (DOC) were determined in the leaf washes; climatic parameters (temperature and humidity) are averages for June 2010 .

graphical distance between communities and that most of this correlation is driven by betaproteobacteria.

\section{MATERIALS AND METHODS}

Sampling. To enable a robust biogeographical analysis of the patterns of bacterial colonization on Tamarix aphylla leaves, we sampled leaf specimens of this tree along a $500-\mathrm{km}$ transect in the southwestern United States. The collection sites were identified from previously recorded $T$. aphylla establishments (data provided by the participants of the Consortium of California Herbaria; ucjeps.berkeley.edu/consortium/) and selected for their relatively uniform climatic conditions (Table 1) and geographic distribution throughout the Sonoran Desert in California and Arizona (Fig. 1). The mean maximum temperatures at the time of sampling (June 2010) were in the range of 38 to $40^{\circ} \mathrm{C}$. Tamarix aphylla trees are not native to the area; they were planted decades ago for decorative purposes or as windbreaks (6).

Five sites were selected and designated A to E (Fig. 1); two trees were sampled within $1 \mathrm{~km}$ of each other at each site. Exact GPS coordinates were recorded for each location. Branches were collected in sterile paper bags and processed within a few hours. Leaf tissue samples, $40 \mathrm{~g}$ each, were immersed in $40 \mathrm{ml}$ sterile $0.1 \mathrm{M} \mathrm{KPO}_{4}(\mathrm{pH} 7)$, sonicated for $2 \mathrm{~min}$ in an ultrasonic cleaning bath, and then vortexed for $15 \mathrm{~s}$. The process was repeated 6 times over a period of $30 \mathrm{~min}$. The leaf wash was decanted, and bacteria were recovered on $0.45-\mu \mathrm{m}$ cellulose nitrate filters by vacuum filtration. The filters were halved and immediately frozen for transit on dry ice.

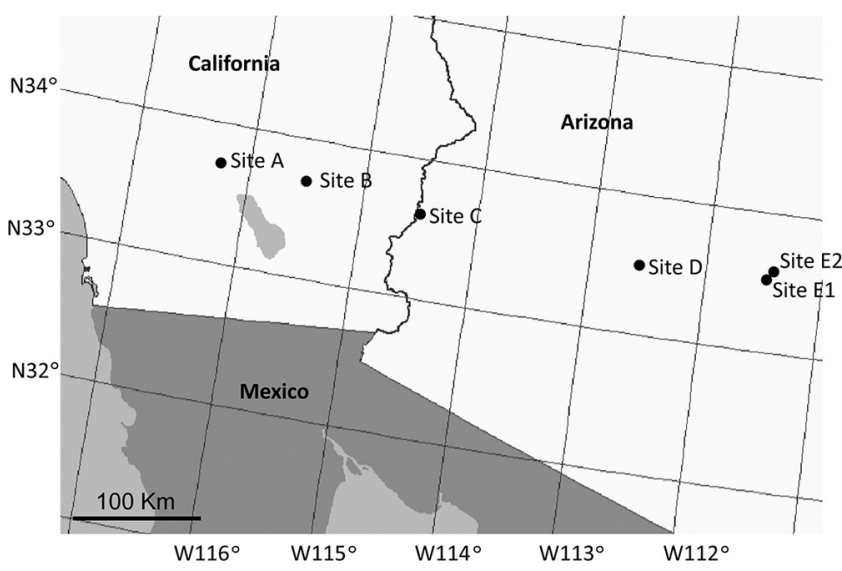

FIG 1 Sampling site locations in the Sonoran Desert, Southwestern United States. The map was generated using the Map Maker application from the National Atlas of the United States website.
DNA extraction. Upon arrival at the laboratory, DNA was extracted from the frozen filters (PowerSoil kit; MoBio, Carlsbad, CA, USA). Each filter half was analyzed separately to control for contamination and PCR bias. Data from both filter halves were later combined to form one data set per sample.

Analysis of the leaf environment chemistry. Leaf washes were also collected for chemical analysis. For each tree sample, $1 \mathrm{~g}$ of leaf tissue was washed and vortexed twice for $30 \mathrm{~min}$ in $10 \mathrm{ml}$ sterile water. One leaf wash was immediately filter sterilized for electrical conductivity and $\mathrm{pH}$ measurements and then frozen on dry ice, while the other was decanted, filter sterilized, adjusted to $\mathrm{pH} 2$ for analysis of dissolved organic carbon (DOC), and then frozen. Electrical conductivity (EC) was measured using a conductivity meter (S30 Seveneasy Conductivity; Mettler, Toledo, $\mathrm{OH}$ ). The correlation between EC values (in $\mathrm{mS} / \mathrm{cm}$ ) and the concentration of $\mathrm{Na}^{+}$ions in the leaf wash (in $\mathrm{mg} /$ liter) was established using an inductively coupled plasma optic emission spectrometer (ICP-OES) (Optima 3000; Perkin Elmer, Waltham, MA) and was found to be linear $(\mathrm{EC}=3.4$. $\left.\left[\mathrm{Na}^{+}\right]-10.6 ; R^{2}=0.99\right)$. $\mathrm{pH}$ was determined using a $\mathrm{pH}$ meter equipped with a combination glass electrode (model 420 ThermoOrion; Orion). DOC was determined using a FormacsHT high-temperature total organic carbon analyzer (Skalar Analytical B. V., Breda, Netherlands) following the removal of inorganic carbon by lowering the $\mathrm{pH}$ to $<2.0$.

Climatic data for June 2010 were collected from Weather Underground (wunderground.com). The data used in the analysis included average minimum temperatures and average maximum humidity. There was no recorded precipitation in any of the sites during that month.

$16 S$ rRNA gene pyrosequencing. Details of this method have been described elsewhere $(4,16,32)$. DNA samples were PCR amplified using primers flanking the bacterial V4-V6 hypervariable regions in the smallsubunit rRNA gene. These amplicons were pyrosequenced using the $\mathrm{Ge}-$ nome Sequencer FLX System (Roche) with Titanium reagents/protocols. To decrease error rates, the sequencing output was passed through several quality filters in addition to the ones built into the system (15). Reads with ambiguous nucleotides, reads lacking an exact match to the sample key or to the proximal $1064 \mathrm{R}$ primer sequence, and reads with more than 3 mismatches to the 565F trimming anchor were omitted from analyses.

Phylogenetic and statistical analysis. Chimeric sequences were evaluated using UChime (USearch with the - uchime option) (7). To ensure optimal chimera identification, we ran UChime with the "reference database" option (similar to Chimera Slayer [12]) using the rRNA 16S Gold fasta file of chimera-checked reference sequences, as well as with the de novo option (default options and abskew of 5; similar to Perseus [27]). Reads identified as chimeras with either option were excluded from the subsequent operational taxonomic unit (OTU) analysis. Sequences were assigned taxonomic names using Global Alignment for Sequence Taxonomy (GAST) (16) and were subsequently separated into seven subsets for clustering according to their assignment to phylum or proteobacterial 
class (Alphaproteobacteria, Betaproteobacteria, Gammaprotobacteria, Firmicutes, Actinobacteria, Bacteroidetes, and all other remaining sequences). Sequences were grouped into $3 \%$ OTU clusters by the single-linkage preclustering (SLP) method (17), designed to minimize OTU inflation caused by sequencing errors, by using a pairwise alignment method and a $2 \%$ single-linkage preclustering methodology; this was followed by an average-linkage clustering based on the same pairwise alignments (17, 27). The same subsets were maintained to facilitate downstream analyses.

Richness estimators were calculated using MOTHUR (31). $\beta$-Diversity was analyzed using the VEGAN package in $\mathrm{R}$ (26). A similarity matrix was generated using a probabilistic distance metric referred to hereinafter as the "Chao" metric (2). This metric was designed to account for unseen shared species in undersampled data that have many rare species and unequal sample sizes, typical of microbial pyrosequencing data. The validity of the measurement of $\beta$-diversity based on OTU clusters was tested by comparing this sequence-based pairwise dissimilarity to a pairwise dissimilarity calculated using the GAST taxonomic assignments. The two measurement methods were found to be nearly equivalent, with the $3 \%$ OTU-based dissimilarity approximately $10 \%$ higher than the taxonomicbased one $\left(\right.$ dissimilarity $_{3 \% \text { ОтU }}=1.1 \cdot$ dissimilarity $_{\text {genera }} ; R^{2}=0.84$; see Fig. S1 in the supplemental material).

We have correlated these dissimilarity matrices with environmental parameters (Table 1) that included climatic information (temperature and humidity), leaf chemistry data (salinity, $\mathrm{pH}$, and DOC), and geographic location (longitude, latitude, and altitude). Comparisons were carried out using environmentally fitted, nonmetric multidimensional scaling (NMDS) plots (R VEGAN package) $(24,26)$. The statistical significance of the relationship between (i) species dissimilarity and geographic distance or (ii) species dissimilarity and difference in environmental parameters was tested by partial Mantel tests (R VEGAN package) $(20,26)$. This tool, selected because the dissimilarity matrix values were not necessarily independent, was employed to isolate the effects of correlated parameters from one another. $P$ values were calculated using 100,000 permutations on rows and columns of dissimilarity matrices.

Sequence Read Archive accession number. All sequences have been deposited in the NCBI Sequence Read Archive (project ID SRP005695).

\section{RESULTS}

Bacterial diversity of the Tamarix phyllosphere metacommunity. After applying all quality filters, a total of 163,895 V4-V6 16S rRNA gene sequences, 481 nucleotides in length, have been obtained from the metacommunity of epiphytic bacteria on Sonoran Desert Tamarix leaves. The reads clustered into 29,048 OTUs, based upon $3 \%$ sequence dissimilarity. Proteobacteria were the most abundant, accounting for 65,944 16S rRNA gene sequences. Of the proteobacteria, the gammaproteobacteria contributed most to the diversity, with over 10,000 OTUs assigned to this phylum. The Chaol species richness estimator was calculated using a subset of 8,700 16S rRNA gene sequences for each site (Table 2). Estimated richness ranged between 6,862 OTUs for site B1 and 21,704 OTUs for site A1. Of the environmental parameters tested, only altitude appeared to explain some of the variation in species richness (Spearman's $S=22.8 ; P=0.004$ ).

Taxonomic assignment of the reads identified 1,010 genera, only 22 of which were found at a relative abundance greater than $5 \%$ in at least one sample (Fig. 2; also see Fig. S3 in the supplemental material). For the sake of clarity, only these genera are included in Fig. 2. The majority of these abundant genera were shared by all samples. One sample that stands out as a clear outlier is D1, which appears to be dominated by the genus Bartonella, comprising over $70 \%$ of the $16 \mathrm{~S}$ rRNA gene sequences from this site while undetected in all other locations. This organism, a known intracellular pathogen (37) of mammals, is thus very likely not a part of the
TABLE 2 Species richness estimation for different sites

\begin{tabular}{ll}
\hline Site & Chaol species richness estimation \\
\hline A1 & 21,704 \\
A2 & 18,058 \\
B1 & 6,862 \\
B2 & 11,446 \\
C1 & 9,173 \\
C2 & 8,023 \\
D2 & 7,571 \\
E1 & 11,692 \\
E2 & 11,843 \\
\hline
\end{tabular}

${ }^{a}$ Chaol estimator was calculated for a randomized subset of 8,700 reads per site.

native microbial phyllosphere population. As site D1 was also identified in a preliminary NMDS analysis as an extreme outlier, it was omitted from further analyses.

The Sonoran Desert phyllosphere community resembles that of Tamarix from other arid regions. To place our results in a global context, a comparison of community composition was performed, combining the current sampling effort with previously acquired data from three different Tamarix tree species in sites by the Dead Sea, the Mediterranean, and in the Negev desert, Israel (9). As the samples from Israeli sites were obtained using a different primer set than the one used here, the community comparison was performed using taxonomic assignment (GAST) (16) and not by OTU clustering. This comparison of Tamarix phyllosphere biodiversity reveals that the bacterial community of the Tamarix phyllosphere has a relatively similar composition in trees from arid regions in both Israel and the United States, as reflected by their relative branch clustering shown in in Fig. 3. The U.S. Sonoran communities are clearly more similar to samples from the Dead Sea region in Israel than to the wetter "Mediterranean" trees, which include the samples from the Negev and from the U.S. island of Martha's Vineyard as outliers. Since community composition differed significantly between different climatic zones in close geographic proximity (Dead Sea versus Mediterranean), regional climate appears to play an important role in the assembly of these communities. Therefore, this global community comparison underscores the importance of our efforts to isolate the distance variable by controlling for climatic variability over our sampling transect.

Similarity in community composition depends upon both geographical distance and environmental parameters. To visualize the similarity between sites and its correlation with environmental parameters, NMDS analysis (Fig. 4, left) was performed. The relative positioning of sites in this plot, as determined by community similarity, generally corresponded to the geographical gradient. The gradient in the different factors characterizing the sampling sites is represented here by vectors, their direction highlighting the mean direction of the gradient and their length the magnitude of correlation between the gradient and community dissimilarity. This vector fitting revealed that geographical distance indeed plays a major role, with longitude being the most significant driver of community dissimilarity. Salinity and humidity were also found to have a significant, albeit weaker, effect on community dissimilarity, even under the relatively uniform climatic conditions prevailing throughout the sampled region. Interestingly, $\mathrm{pH}$, an abiotic factor known to affect bacterial diversity in soils $(8,19)$, was the only measured abiotic factor that did 


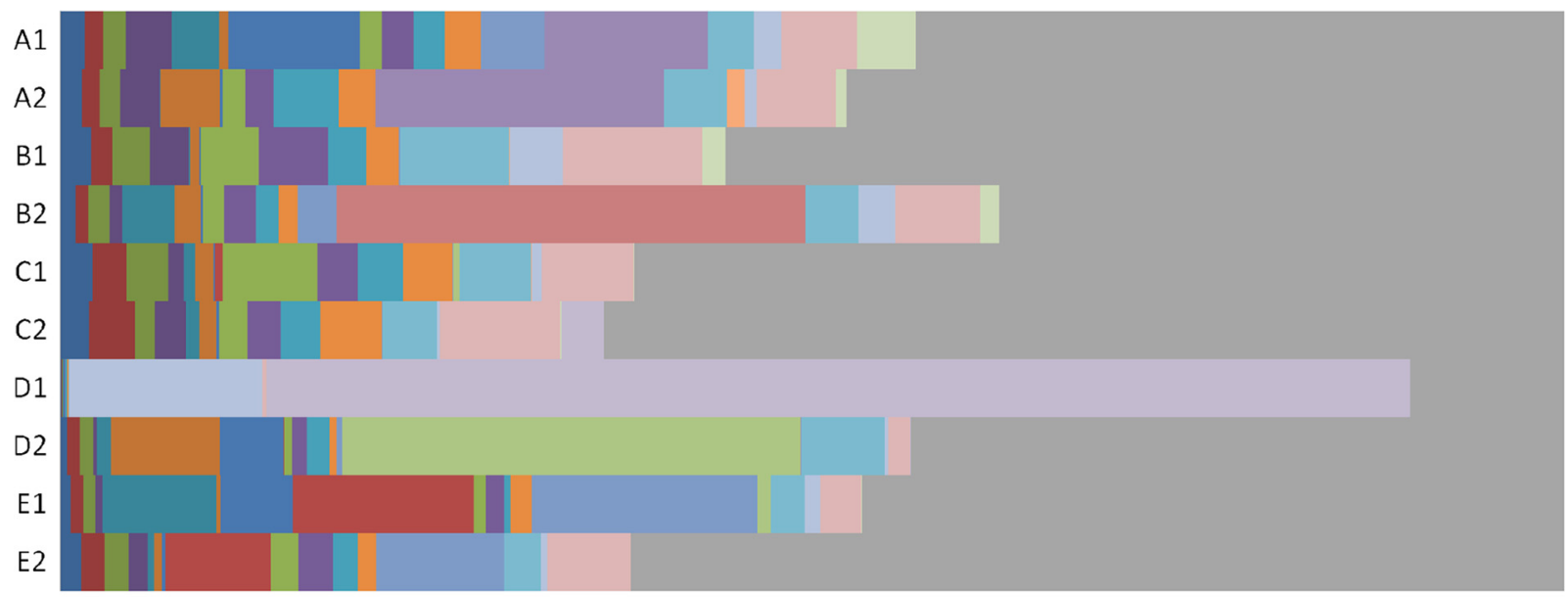

- Actinomycetales;Cellulomonadaceae;Cellulomonas nhizobiales;Bradyrhizobiaceae;Balneimonas

- Enterobacteriales;Enterobacteriaceae;genus_NA

- Pseudomonadales;Moraxellaceae;Acinetobacter - Rhodospirillales;Rhodospirillaceae;Skermanella

- Bacillales;Planococcaceae;Planococcus

- Oceanospirillales;Halomonadaceae;Zymobacter

Rhodospirillales;Acetobacteraceae;Saccharibacter

- Actinomycetales; Micrococcaceae; Kocuria

- Rhodospirillales;Acetobacteraceae;genus_NA

Gammaproteobacteria;orderx_NA;family_NA;genus_NA

- rare genera $(<5 \%)$
Rhodobacterales; Rhodobacteraceae;Rubellimicrobium

- Actinomycetales;Geodermatophilaceae;Modestobacter

- Flavobacteriales;Flavobacteriaceae;Salinimicrobium

- Enterobacteriales;Enterobacteriaceae;Serratia

Actinomycetales;Geodermatophilaceae;Blastococcus

- Bacillales;Bacillaceae;Bacillus

- Enterobacteriales;Enterobacteriaceae;Klebsiella

- Flavobacteriales;Blattabacteriaceae;genus_NA

Burkholderiales;Burkholderiaceae;Burkholderia

Actinomycetales;Geodermatophilaceae;Geodermatophilus

- Rhizobiales;Bartonellaceae;Bartonella

FIG 2 Taxonomic assignment of $16 \mathrm{~S}$ rRNA gene sequences to genera in each of the T. aphylla leaf samples. Only the 22 genera found at a relative abundance greater than $5 \%$ are included by name. All other genera are grouped into the gray areas on the right hand side of each of the bars, completing the count to $100 \%$ of the number of identified genera. Taxonomic names ending in "_NA" have no assignment of affiliation at the relevant taxonomic level.

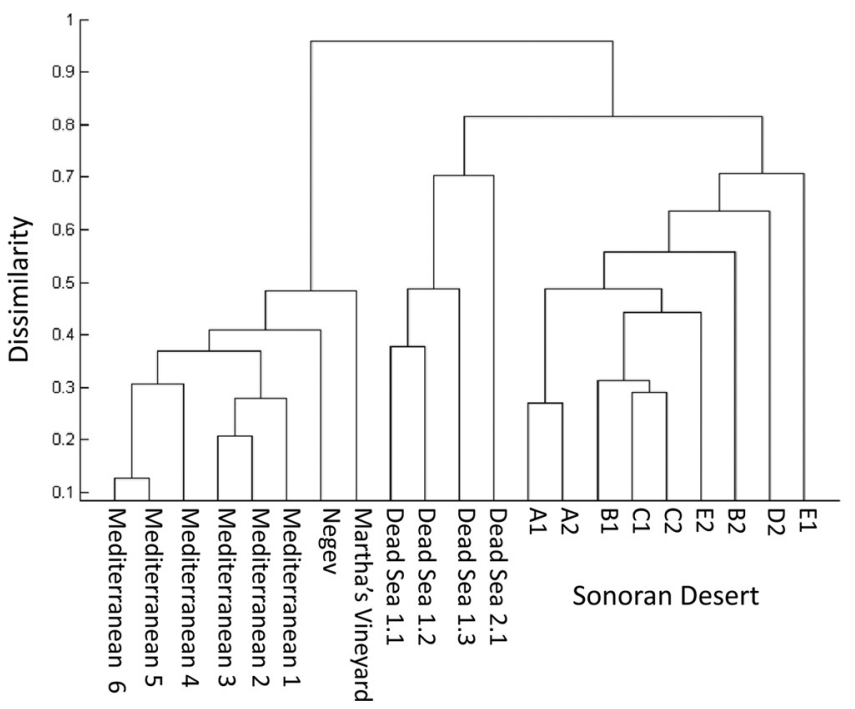

FIG 3 Unweighted-pair group method using average linkages (UPGMA) dendrogram depicting Chao pairwise dissimilarities (based on taxonomic affiliations) between a global array of samples. The samples cluster first according to climate (temperate sites on the left, extreme desert sites on the right), and then by location (sites in Israel in the left subclusters, U.S. samples in the right subclusters). not appear to be correlated with the community composition of any of the tested phyla.

Although the sampling scheme was designed to minimize environmental variability, the NMDS results nevertheless suggested some correlation between geographical distance and certain environmental variables. Indeed, the combined dissimilarity in maximum daily humidity, minimal daily temperature, and leaf salinity (calculated using the Gower dissimilarity metric) (10) was linearly correlated with geographical distance (see Fig. S2 in the supplemental material). To separate the effect of geography from the environmental factors that may be correlated with it, we subjected the data to partial Mantel tests, designed to measure the correlation between two dissimilarity matrices. The tests were performed with the purpose of establishing a partial correlation between community dissimilarity, geographical distance, and the combined differences in temperature, humidity, and salinity, using both the entire bacterial community data set and taxonomic subsets. Mantel analysis of the entire bacterial community (Fig. 5A and $B)$ revealed no significant correlation for either geographical distance (on top of environmental conditions; $R=0.25, P=0.17$ ) (Fig. 5A) or environmental conditions (on top of geographical distance; $R=0.1$, $P=0.32$ ) (Fig. 5B), signifying that although the effect of geographical distance is stronger, it cannot be separated from the effect of some environmental conditions. When performing this analysis on the taxonomic subsets (adjusting for multiple comparisons), only betaproteobacterial sequences (Fig. 5C and D) exhibited a significant corre- 
Bacteria

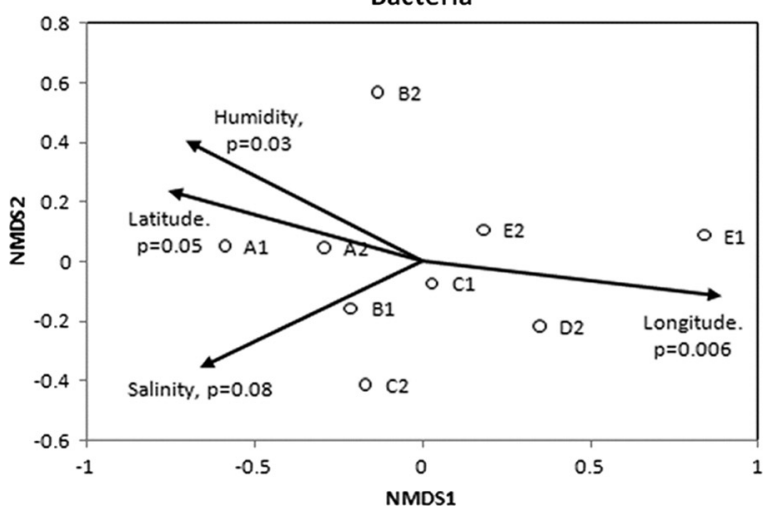

Betaproteobacteria

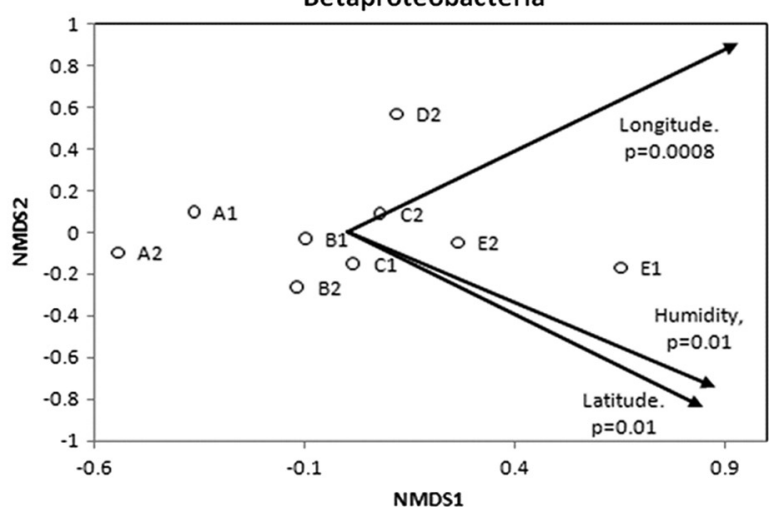

FIG 4 Correlations between environment, geography, and community dissimilarity. Environmentally fitted NMDS plots based on Chao pairwise dissimilarity index for the entire population (left) or for betaproteobacteria only (right). The points indicate the sampling site (as shown in Fig. 1), and the vectors represent the mean direction and strength of correlation of environmental variables. $P$ values noted by the vectors were calculated by permutations of environmental data.

lation with geographical distance, assuming uniform environmental conditions $(R=0.53, P=0.0057)$ (Fig. 5C). A similar correlation was not found with environmental conditions when assuming no geographical distance $(R<0, P=0.78)$ (Fig. 5D), confirming that a significant distance-decay relationship is observed for betaproteobacteria in this metacommunity. An NMDS analysis for the betaproteo- bacteria alone confirms this observation, with a clear east-west gradient manifested along the first axis (Fig. 4B). The trends that appear in Fig. 5 can be compared to the results in Fig. 1 of Martiny et al. (23), displaying the "multiple habitats and provinces" scenario for the entire bacterial population and the "one habitat, multiple provinces" scenario for betaproteobacteria only.
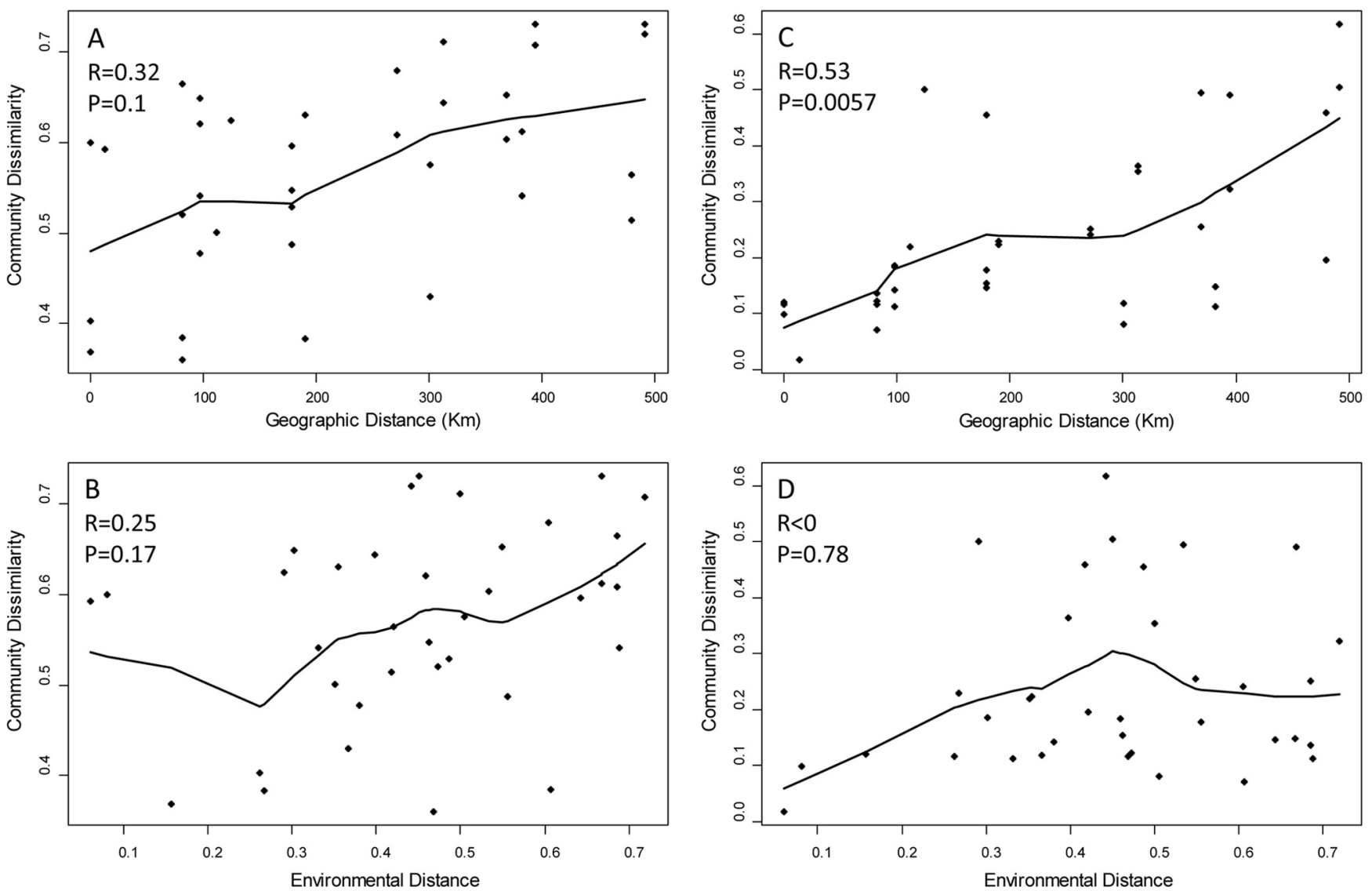

FIG 5 Correlation between geographical distance (A, C), environmental distance (B, D), and Chao pairwise dissimilarity index for the entire population (A, B) or for betaproteobacteria only $(\mathrm{C}, \mathrm{D})$. Locally weighted polynomial regression lines (3) are shown. The significance of correlation was tested using partial Mantel tests. 


\section{DISCUSSION}

Our results demonstrate that for some bacterial taxa, community similarity declines significantly with geographic distance. The decay of community similarity with distance is a prediction of neutral ecological theories (14), corroborated in numerous macroorganism studies $(25,33,34)$. The presence of the same effect in bacteria, albeit with weaker rates, emphasizes the importance of dispersal in bacterial community assembly.

The scale at which microbial diversity is structured can vary greatly in time and space. Green and Bohannan (11) have reviewed a number of studies that tested the effect of geographic distance on microbial community similarity in different taxa at spatial scales that varied from a few centimeters to thousands of kilometers; the mixed results they have noted led them to recommend the use of more robust statistical approaches. Such methods were applied for bacterial communities in soil (8), in salt-marsh sediments (13), and in tree holes (1), all concluding that the effect of geographic distance is overshadowed by the effect of environmental heterogeneity. Interestingly, Horner-Devine et al. (13) found no effect of distance on betaproteobacterial community similarity, in contrast to that demonstrated here on Tamarix.

Contradictory results have also been obtained for biogeographical studies of phyllosphere bacteria. Redford et al. (30) demonstrated that pine trees in Australia and the United States harbor similar bacterial communities. Knief et al. (18), in contrast, have shown that geographic location may play a more important role than host tree species in determining its microbial epibiome composition. These reports, however, did not control for variation in environmental conditions between sampling sites. In the present communication, we show that a phenomenon similar to the one observed by Redford et al. for Pinus ponderosa leaves occurs on Tamarix leaves, as phyllosphere populations from Israeli and U.S. deserts are similar (Fig. 4). However, by selecting sampling sites to minimize differences in environmental variability and by performing multivariate statistical analysis to account for the small variation in environments, we were able to isolate and quantify the effect of distance on bacterial community dissimilarity in the phyllosphere.

Multivariate analysis of the effects of environmental and geographic parameters on bacterial community composition revealed that geographic location, salinity, and humidity differentially affected members of different phyla. Interestingly, we did not observe any influence of $\mathrm{pH}$, previously noted as a strong determinant in other environments $(8,19)$. However, where $\mathrm{pH}$ has been noted to have a strong effect, this occurred across a broader $\mathrm{pH}$ range ( $\mathrm{pH} 3$ to 9 ) than the exclusively alkaline conditions ( $\mathrm{pH} 7.8$ to 10.1) encountered in this study. Because the $T$. aphylla phyllosphere is already alkaline, it may select for taxa most suited to alkaline growth conditions, and the further contribution of $\mathrm{pH}$ to bacterial diversity may thus be small.

While similarity analyses encompassing the entire bacterial community are highly useful, they require viewing the microbial community as a whole, a view that ignores, for instance, that this diverse assemblage of species may span several trophic levels and different persistence strategies. This observed decline in community similarity is not uniform for all bacteria, and some taxa appear to be more limited in dispersal than others, demonstrating that the analysis of $\beta$-diversity of microbial communities as a whole, often adopted due to technical constraints, may mask important pro- cesses seen in a subset of the community. That being said, our finding that only betaproteobacteria are distance dependent in this environment cannot at present be correlated with a certain unique trait that is shared by members of this class. Furthermore, we cannot rule out the notion that the measured distance decay may in fact be the result of an unmeasured environmental variable that is correlated with geographic location. These points require further investigation, possibly via an experimental scheme similar to that presented by Bell (1).

Tamarix leaves are an extreme environment, with high salinity and alkalinity, but also are characterized by elevated local concentrations of organic carbon and moisture. This ensemble of factors is most likely not otherwise encountered in the surrounding environment. Tamarix trees are found in a wide variety of climates, enabling the study of a variety of globally important environmental parameters, such as salinity, water availability, temperature, and altitude, and their effects on bacterial community composition. The Tamarix phyllosphere, therefore, is an attractive model for studying the interplay of global and local factors in the determination of microbial community structure.

\section{ACKNOWLEDGMENTS}

The research was supported in part by the United States-Israel Binational Science Foundation under grant number 2006324 to S.B. and S.E.L. and grant number 2010262 to S.B. and A.F.P. O.M.F. and S.B. are indebted to the Gruss-Lipper Family Foundation for a summer research fellowship at the Marine Biology Laboratory (MBL; Woods Hole, MA) that supported pyrosequencing and data analysis. S.M.H. was supported by a grant from the Sloan Foundation.

We are grateful to the staff and scientists of the Josephine Bay Paul Center in Comparative Molecular Biology and Evolution at the MBL for their help during and after the time of the summer research fellowship. We thank Lorelei Bornfleth for assistance with leaf collections. Earlier support of the Tamarix phyllosphere biodiversity study by the Bridging the Rift Foundation is also gratefully acknowledged, as are the most-helpful comments of Ronen Kadmon, the Hebrew University of Jerusalem.

\section{REFERENCES}

1. Bell T. 2010. Experimental tests of the bacterial distance-decay relationship. ISME J. 4:1357-1365.

2. Chao A, Chazdon RL, Colwell RK, Tsung-Jen S. 2005. A new statistical approach for assessing similarity of species composition with incidence and abundance data. Ecol. Lett. 8:148-159.

3. Cleveland WS. 1981. LOWESS: a program for smoothing scatterplots by robust locally weighted regression. Am. Stat. 35:54.

4. Dethlefsen L, Huse S, Sogin ML, Relman DA. 2008. The pervasive effects of an antibiotic on the human gut microbiota, as revealed by deep $16 \mathrm{~S}$ rRNA sequencing. PLoS Biol. 6(11):e280. doi:10.1371/journal. pbio. 0060280 .

5. de Wit R, Bouvier T. 2006. "Everything is everywhere, but the environment selects"; what did Baas Becking and Beijerinck really say? Environ. Microbiol. 8:755-758.

6. Di Tomaso, JM. 1998 Impact, biology, and ecology of saltcedar. Tamarix spp. in the Southwestern United States. Weed Technol. 12:326-336.

7. Edgar RC. 2010. Search and clustering orders of magnitude faster than BLAST. Bioinformatics 26(19):2460-2461. doi:10.1093/bioinformatics/ btq461.

8. Fierer N, Jackson RB. 2006. The diversity and biogeography of soil bacterial communities. Proc. Natl. Acad. Sci. U. S. A. 103:626-631.

9. Finkel O, Burch AY, Lindow SE, Post AE, Belkin S. 2011. Geographical location determines the population structure in phyllosphere microbial communities of a salt-excreting desert tree. Appl. Environ. Microbiol. 77:7647-7655.

10. Gower JC. 1971. A general coefficient of similarity and some of its properties. Biometrics 27:623-637.

11. Green J, Bohannan BJM. 2006. Spatial scaling of microbial biodiversity. Trends Ecol. Evol. 21:501-507. 
12. Haas BJ, et al. 2011. Chimeric $16 \mathrm{~S}$ rRNA sequence formation and detection in Sanger and 454-pyrosequenced PCR amplicons. Genome Res. 21: 494-504.

13. Horner-Devine MC, Lag M, Hughes JB, Bohannan BJM. 2004. A taxaarea relationship for bacteria. Nature 432:750-753.

14. Hubbell SP. 2001. The unified neutral theory of biodiversity and biogeography. Princeton University Press, Princeton, NJ.

15. Huse SM, Huber JA, Morrison HG, Sogin ML, Welch DM. 2007. Accuracy and quality of massively parallel DNA pyrosequencing. Genome Biol. 8(7):R143. doi:10.1186/gb-2007-8-7-r143.

16. Huse SM, et al. 2008. Exploring microbial diversity and taxonomy using SSU rRNA hypervariable tag sequencing. PLoS Genet. 4:e1000255. doi: 10.1371/journal.pgen.1000255.

17. Huse SM, Welch DM, Morrison HG, Sogin ML. 2010. Ironing out the wrinkles in the rare biosphere through improved OTU clustering. Environ. Microbiol. 12:1889-1898.

18. Knief C, Ramette A, Frances L, Alonso-Blanco C, Vorholt JA. 2010. Site and plant species are important determinants of the Methylobacterium community composition in the plant phyllosphere. ISME J. 4:719-728.

19. Lauber CL, Hamady M, Knight R, Fierer N. 2009. Pyrosequencing-based assessment of soil $\mathrm{pH}$ as a predictor of soil bacterial community structure at the continental scale. Appl. Environ. Microbiol. 75:5111-5120.

20. Legendre P, Legendre L. 1998. Numerical ecology, 2nd English ed. Elsevier, Amsterdam, Netherlands.

21. Leibold MA, et al. 2004. The metacommunity concept: a framework for multi-scale community ecology. Ecol. Lett. 7:601-613.

22. MacArthur RH, Wilson EO. 1967. The theory of island biogeography: Princeton University Press, Princeton, NJ.

23. Martiny JBH, et al. 2006. Microbial biogeography: putting microorganisms on the map. Nat. Rev. Microbiol. 4:102-112.

24. Minchin PR. 1987. An evaluation of relative robustness of techniques for ecological ordinations. Vegetation 71:145-156.
25. Necola JC, White PS. 1999. The distance decay of similarity in biogeography and ecology. J. Biogeogr. 26:867-878.

26. Oksanen J, et al. 2008. Vegan: community ecology package. R package version 1.15-1. http://cran.r-project.org/; http://vegan.r-forge.r-project .org/.

27. Quince C, Lanzen A, Davenport R, Turnbaugh P. 2011. Removing noise from pyrosequenced amplicons. BMC Bioinformatics 12:38. doi:10.1186/ 1471-2105-12-38

28. Qvit-Raz N, Jurkevitch E, Belkin S. 2008. Drop-size soda lakes: transient microbial habitats on a salt-secreting desert tree. Genetics 178:1615-1622.

29. Qvit-Raz N, et al. 2012. Biogeographical diversity of leaf-associated microbial communities from salt-secreting Tamarix trees of the Dead Sea region. Res. Microbiol. 163:142-150.

30. Redford AJ, Bowers RM, Knight R, Linhart Y, Fierer N. 2010. The ecology of the phyllosphere: geographic and phylogenetic variability in the distribution of bacteria on tree leaves. Environ. Microbiol. 12:2885-2893.

31. Schloss PD, et al. 2009. Introducing mothur: open source, platformindependent, community-supported software for describing and comparing microbial communities. Appl. Environ. Microbiol. 75:7537-7541.

32. Sogin ML, et al. 2006. Microbial diversity in the deep sea and the underexplored "rare biosphere." Proc. Natl. Acad. Sci. U. S. A. 103:1211512120.

33. Soininen J, McDonald N, Hillebrand H. 2007. The distance decay of similarity in ecological communities. Ecography 30:3-12.

34. Steinitz O, Heller J, Tsoar A, Rotem D, Kadmon R. 2006. Environment, dispersal and patterns of species similarity. J. Biogeogr. 33:1044-1054.

35. Waisel Y. 1961. Ecological studies on Tamarix aphylla (L.) Karst. Plant Soil 13:356-364.

36. Waisel Y. 1991. The glands of Tamarix aphylla: a system for salt recretion or for carbon concentration? Physiol. Plant. 83:506-510.

37. Zeaiter Z, Fournier PE, Ogata H, Raoult D. 2002. Phylogenetic classification of Bartonella species by comparing groEL sequences. Int. J. Syst. Evol. Microbiol. 52:165-171. 